\title{
A STUDY OF NEPHELAUXETIC RATIO OF RARE-EARTH COMPLEXES (NEODYMIUM) IN DIFFERENT AMINO-ACIDS
}

\section{DEEPMALA}

Head, Department of Physics, Sophia Girls College, Ajmer, Rajasthan, India

\begin{abstract}
Among metals, the rare-earth elements form an outstanding and unique group. The element of the first series that is from Cerium to Lutetium $(58 \mathrm{Ce}$ to $71 \mathrm{Lu})$ which form a part of the sixth period are called lanthanides. These are called rare-earth elements because of the very small amount of it. The Configuration of rare-earths ion is given by (1s22s2 2p63s23p63d104s24p64d105s25p6)4f n5d16s 2 and the value of $n$ varies from one to fourteen.Ternary complexes of Neodymium with amino acids that is Arginine, Threonine or Leucine as primary ligand and Urea as secondary ligands are prepared in 1:1:2 ratio. The spectra of these complexes are recorded by UV-VIS spectrophotometer, LABINDIA 3000+. The value of different parameters- Racah parameter (E1, E2, E3), Slater-Condon parameters(F2,F4,F6) and Lande's parameter are calculated. The effect of various amino acids on the position and intensity has been discussed in terms of bonding parameter. After calculation of these Slater-Condon parameters, Nephelauxetic Ratio is calculated this is directly related to types of bonding.

KEYWORDS: Neodymium, Slater-Condon Parameters, Nephelauxetic Ratio \& UV Spectrophotometer
\end{abstract}

Received: Jan 01, 2019; Accepted: Jan 21, 2019; Published: Feb 06, 2019; Paper Id.: IJPRJUN20191

\section{INTRODUCTION}

Neodymium consists of two Greek words that is Neos meant new and didymos meant twins. Now a days, Neodymium is mainly used in lasers, ceramic glasses and ceramic capacitors[1-20]. In case of ternary complexes of Neodymium, the colour of it is reddish purple because of $\mathrm{Nd}^{+3}$. It can easily oxidise in air and it is one of the most reactive elements. In the pure form of Neodymium that is in the Free State its spectra lies in infrared red, visible and ultraviolet region. In ternary complexes of Neodymium, some peaks are displaced from its original positions. Neodymium peak, which shows maxima is ${ }^{4} \mathrm{G}_{5 / 2}$. This represents a hypersensitive position of transition.

Hypersensitive bands are very sensitive to the change in environments. Different researchers gave different explanations of these bands. According to Judd[10,11], these bands are due to spherical Harmonics. But according to Choppin hypersensitivity is due to inclusion of Covalency within the framework of a vibronic mechanism. The different modes by which electronic transitions take place are

- Electric dipole

- Magnetic dipole

- Electric Quadrupole

In case of rare earth complexes, observed intensities of the spectral lines are too large and cannot be explained on the basis of magnetic dipole and electric quadrupole interactions. Induced electric dipole transitions are mainly responsible for the occurrence of these lines. Induced electric dipole transition is strongest in three. 
Hypersensitive transitions obey the selection rule of quadrupole transitions and therefore known as Pseudo-quadrupole transitions.

\section{EXPERIMENTAL DETAILS}

In the present study, one Rare-earth metal Neodymium is taken. The complexes of Neodymium are prepared by taking one of the Amino-acid that is Arginine, Leucine, Threonine as primary ligand and urea as secondary ligand mixed in 1:1:2 ratio. Their ternary complexes are studied in solvent form. The calculated energy levels corresponding to different peaks of Neodymium Complexes are compared with the experimentally observed values. The energy level values change due to change in ligands or change in solvents. All reagents used in this study are taken of standard purity. In this work all chemicals are from C.D.H Company. The solution is stirred for half an hour with the magnetic stirrer method. The formation of complex is also checked by infrared spectra in Chemistry lab of S.P.C Government College, Ajmer. The absorption spectra of the complexes are recorded with UV-VIS Spectrophotometer LABINDA $3000^{+}$. Peaks of these absorption spectra are recorded in between 400nm to 800nm (Visible Range).

\section{PARAMETERS}

\section{Slater-Condon parameters, Lande's Parameters and Racah Parameters}

Change in environment of rare-earth ions sometimes doesn't change the position of energy levels too much. This causes only a slight change in various parameters [2]. It is very difficult to solve these parameters using the method of diagonalisation because in some cases, number of levels are higher than the number of parameters. Various parameters like Slater-Condon, Lande's and Racah (Energy level parameter) on complexation as compared to free ion reveals, that on complexation there is change in symmetry around the metal ion, types of bonding between metal and ligands etc.

When there is formation of ternary complexes of Neodymium with amino-acids then red shift occurs. On complexation, there is a very small change in F parameters and Lande's parameter. After the formation of complex these two parameters decrease slightly. The decrease in value of Lande's parameter is more than Slater-Condon parameters. This signifies that ligands affect spin-orbit coupling more than the electrostatic repulsion. Columbic and spin-orbit parameter decreases and there is expansion of the central metal ion on complexation. Lanthanide complexes have high coordination numbers. Bonding in rare-earth complexes is weaker than in the $3 \mathrm{~d}$ complexes. Bonding parameter $\mathrm{b}^{1 / 2}$ expresses bond strength of rare-earth complexes. When we calculate parameters, we assume $4 \mathrm{f}$ radial eigen function to be hydrogenic. This reduces the number of radial integral three to one. The energy levels of various transitions are experimentally as well as theoretically calculated by using the Taylor Series expansion.

$$
\begin{aligned}
& \mathrm{E}_{\mathrm{j}}=\mathrm{E}_{\mathrm{oj}}+\sum_{\mathrm{K}=2,4,6} \frac{\partial \mathrm{E}_{\mathrm{j}}}{\partial \mathrm{F}_{\mathrm{K}}} \cdot \Delta \mathrm{F}_{\mathrm{K}}+\frac{\partial \mathrm{E}_{\mathrm{j}}}{\partial \zeta_{4 \mathrm{f}}} \Delta \zeta_{4 \mathrm{f}} \\
& \Delta \mathrm{E}_{\mathrm{j}}=\sum_{\mathrm{K}=2,4,6} \frac{\partial \mathrm{E}_{\mathrm{j}}}{\partial \mathrm{F}_{\mathrm{K}}} \Delta \mathrm{F}_{\mathrm{K}}+\frac{\partial \mathrm{E}_{\mathrm{j}}^{0}}{\partial \zeta_{4 \mathrm{f}}} \Delta \zeta_{4 \mathrm{f}}
\end{aligned}
$$

The root mean square deviation between theoretical and experimental value of energy are calculated using the relation

$$
=\sum\left[\frac{\left(\mathrm{E}_{\mathrm{Cal}}-\mathrm{E}_{\mathrm{obs}}\right)^{2}}{\mathrm{~N}}\right]^{1 / 2}
$$




\section{Nephelauxetic Ratio, Bonding Parameter and R.M.S. Deviation}

The bonding in rare-earth complexes is weaker than $3 \mathrm{~d}$ orbital's. When rare-earth ternary complexes are formed the electronic repulsion becomes weaker as compared to free ions. This is due to delocalization of molecular orbitals over the ligands and away from the metal. The chemical bond for rare-earth complex is considered to be ionic because of their inert gas electronic configuration. It is due to well shielding of $4 \mathrm{f}$ orbitals. But experimentally, covalency is also observed for some rare-earth complexes. On complexation, there is expansion of $4 \mathrm{f}$ orbital's which can be expressed in terms of Nephelauxetic Ratio ( $\beta$ )[21-27]. Delocalization of molecular orbitals increases separation between electrons and reduces repulsion between electrons. This reduction is expressed in terms of a ratio known as Nephelauxetic ratio. The Greek meaning of this is cloud expanding.

$$
\begin{aligned}
& \beta=F_{k}^{c} / F_{k}^{f} \\
& \text { Where, } \\
& c=\text { complex state } \\
& \mathrm{f}=\text { free ion state } \\
& \text { Bonding parameter is } b^{1 / 2}=[(1-\beta) / 2]^{1 / 2} \\
& \delta=(1-\beta) / \beta
\end{aligned}
$$

\section{OBSERVATION TABLES}

Table 1: Observed and Calculated Energy Levels of Neodymium: Arginine: Urea in the Molar Ratio 1:1:2

\begin{tabular}{|l|c|c|c|c|c|c|c|c|c|c|}
\hline \multicolumn{1}{|c|}{ Levels } & ${ }^{4} \mathbf{F}_{3 / 2}$ & ${ }^{4} \mathbf{F}_{/ 2}$ & ${ }^{4} \mathbf{F}_{7 / 2}$ & ${ }^{4} \mathbf{F}_{9 / 2}$ & ${ }^{4} \mathbf{G}_{5 / 2}$ & ${ }^{4} \mathbf{G}_{72}$ & ${ }^{4} \mathbf{G}_{9 / 2}$ & ${ }^{2} \mathbf{G}_{9 / 2}$ & ${ }^{4} \mathbf{G}_{11 / 2}$ & ${ }^{2} \mathbf{P}_{1 / 2}$ \\
\hline $\begin{array}{l}\text { Observed } \\
\text { energy }\end{array}$ & 11560.69 & 12594.45 & 13513.51 & 14727.54 & 17391.30 & 19193.85 & 19531.25 & 21052.63 & 21691.97 & 23148.14 \\
\hline $\begin{array}{l}\text { Calculated } \\
\text { energy }\end{array}$ & 11557.97 & 12582.87 & 13408.64 & 14778.03 & 17416.35 & 19209.57 & 19607.38 & 21023.08 & 21692.09 & 23151.14 \\
\hline $\begin{array}{l}\text { Change in } \\
\text { energy }\end{array}$ & 2.72 & 11.58 & 104.87 & -50.49 & -25.04 & -15.72 & -76.13 & 29.05 & -0.11 & -2.99 \\
\hline
\end{tabular}

Table 2: Observed and Calculated Energy Levels of

Neodymium: Threonine: Urea in the Molar Ratio 1:1:2

\begin{tabular}{|l|c|c|c|c|c|c|c|c|c|c|}
\hline \multicolumn{1}{|c|}{ Levels } & ${ }^{4} \mathbf{F}_{3 / 2}$ & ${ }^{4} \mathbf{F}_{/ 2}$ & ${ }^{4} \mathbf{F}_{7 / 2}$ & ${ }^{4} \mathbf{F}_{9 / 2}$ & ${ }^{4} \mathbf{G}_{5 / 2}$ & ${ }^{4} \mathbf{G}_{7 / 2}$ & ${ }^{4} \mathbf{G}_{9 / 2}$ & ${ }^{2} \mathbf{G}_{9 / 2}$ & ${ }^{4} \mathbf{G}_{11 / 2}$ & ${ }^{2} \mathbf{P}_{1 / 2}$ \\
\hline $\begin{array}{l}\text { Observed } \\
\text { energy }\end{array}$ & 11560.70 & 12594.46 & 13495.28 & 14727.54 & 17391.30 & 19157.08 & 19531.12 & 21052.63 & 21691.97 & 23201.18 \\
\hline $\begin{array}{l}\text { Calculated } \\
\text { energy }\end{array}$ & 11553.32 & 12577.16 & 13405.88 & 14780.26 & 17402.89 & 19198.21 & 19595.77 & 21019.08 & 21684.93 & 23203.23 \\
\hline $\begin{array}{l}\text { Change in } \\
\text { energy }\end{array}$ & 7.38 & 17.29 & 89.40 & -52.71 & -11.58 & -41.12 & -64.65 & 33.55 & 7.04 & -2.05 \\
\hline
\end{tabular}

Table 3: Observed and Calculated Energy Levels of Neodymium: Leucine: Urea in the Molar Ratio 1:1:2

\begin{tabular}{|l|c|c|c|c|c|c|c|c|c|c|}
\hline \multicolumn{1}{|c|}{ Levels } & ${ }^{4} \mathbf{F}_{3 / 2}$ & ${ }^{4} \mathbf{F}_{/ 2}$ & ${ }^{4} \mathbf{F}_{7 / 2}$ & ${ }^{4} \mathbf{F}_{9 / 2}$ & ${ }^{4} \mathbf{G}_{5 / 2}$ & ${ }^{4} \mathbf{G}_{7 / 2}$ & ${ }^{4} \mathbf{G}_{9 / 2}$ & ${ }^{2} \mathbf{G}_{9 / 2}$ & ${ }^{4} \mathbf{G}_{11 / 2}$ & ${ }^{2} \mathbf{P}_{1 / 2}$ \\
\hline $\begin{array}{l}\text { Observed } \\
\text { energy }\end{array}$ & 11547.34 & 12594.45 & 13495.27 & 14705.88 & 17301.03 & 19157.08 & 19569.47 & 21008.40 & 21691.97 & 23148.14 \\
\hline $\begin{array}{l}\text { Calculate } \\
\text { d energy }\end{array}$ & 11540.57 & 12572.31 & 13402.01 & 14773.33 & 17384.16 & 19171.29 & 19580.88 & 20988.05 & 21683.87 & 23143.70 \\
\hline $\begin{array}{l}\text { Change } \\
\text { in energy }\end{array}$ & 6.76 & 22.13 & 93.26 & -67.44 & -83.12 & -14.20 & $-11 . .19$ & 20.351 & 8.09 & 4.44 \\
\hline
\end{tabular}


Table 4: Energy Parameters and F Parameters

\begin{tabular}{|c|c|c|c|}
\hline & ND:ARG:U & ND:THREO:U & ND:LEUC:U \\
\hline $\mathrm{E}_{1}$ & 5019.81 & 5038.732 & 5015.19 \\
\hline $\mathrm{E}_{2}$ & 25.11 & 25.42 & 25.43 \\
\hline $\mathrm{E}_{3}$ & 496.98 & 496.46 & 495.71 \\
\hline $\mathrm{E}_{1} / \mathrm{E}_{3}$ & 10.100 & 10.14 & 10.116 \\
\hline $\mathrm{E}_{2} / \mathrm{E}_{3}$ & 0.0505 & 0.05 & 0.0513 \\
\hline $\mathrm{F}_{2}$ & 335.19 & 336.55 & 335.84 \\
\hline $\mathrm{F}_{4}$ & 48.60 & 48.30 & 47.93 \\
\hline $\mathrm{F}_{6}$ & 5.23 & 5.31 & 5.27 \\
\hline $\mathrm{Zeta} 4 \mathrm{~F}$ & 871.22 & 872.88 & 876.81 \\
\hline $\mathrm{F}_{4} / \mathrm{F}_{2}$ & 0.145 & 0.143 & 0.142 \\
\hline $\mathrm{F}_{6} / \mathrm{F}_{2}$ & 0.01562 & 0.01578 & 0.01569 \\
\hline
\end{tabular}

Table 5: RMS Deviation, Nephelauxetic Ratio and Bonding Parameter

\begin{tabular}{|l|c|c|c|}
\hline & ND:ARG:U & ND:THREO:U & ND:LEU:U \\
\hline RMS Deviation & 46.057 & 42.7914 & 46.389 \\
\hline Nephelauxetic Ratio & 1.012 & 1.0162 & 1.014 \\
\hline Bonding Parameter & 0.078 & 0.0902 & 0.084 \\
\hline
\end{tabular}

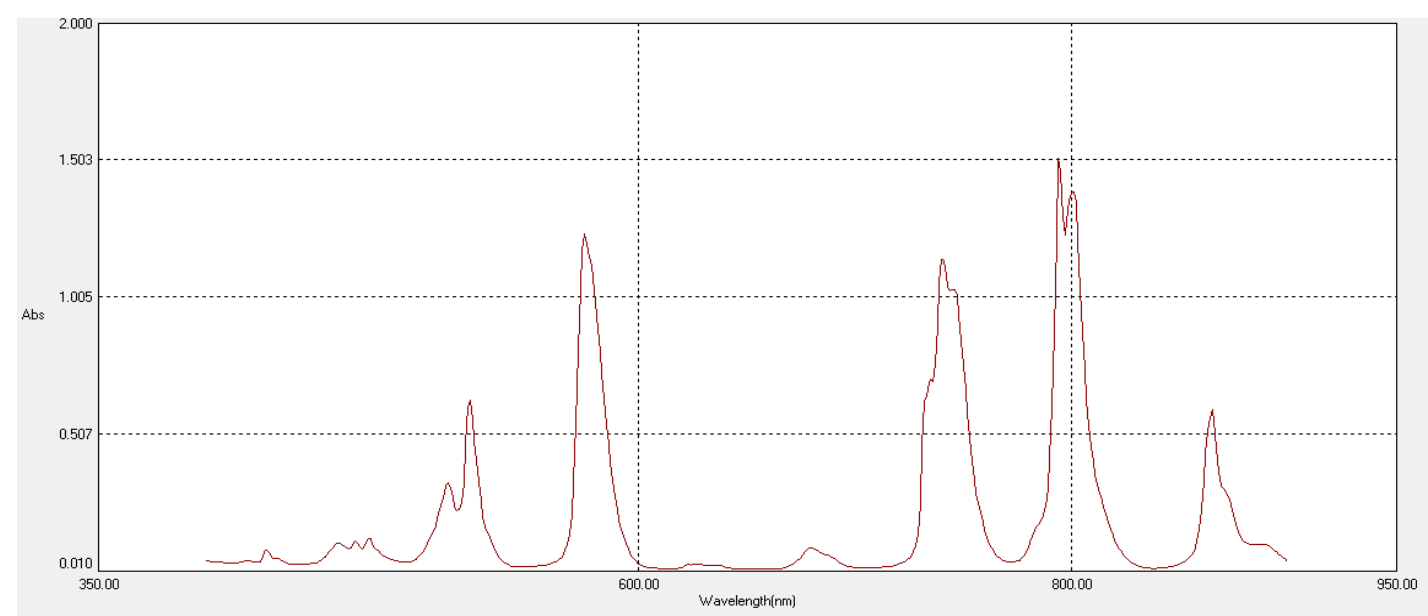

Graph 1: Absorption Spectra of Neodymium: Arginine: Urea in the Molar Ratio 1:1:2



Graph 2: Absorption Spectra of Neodymium: Threonine: Urea in the Molar Ratio 1:1:2 


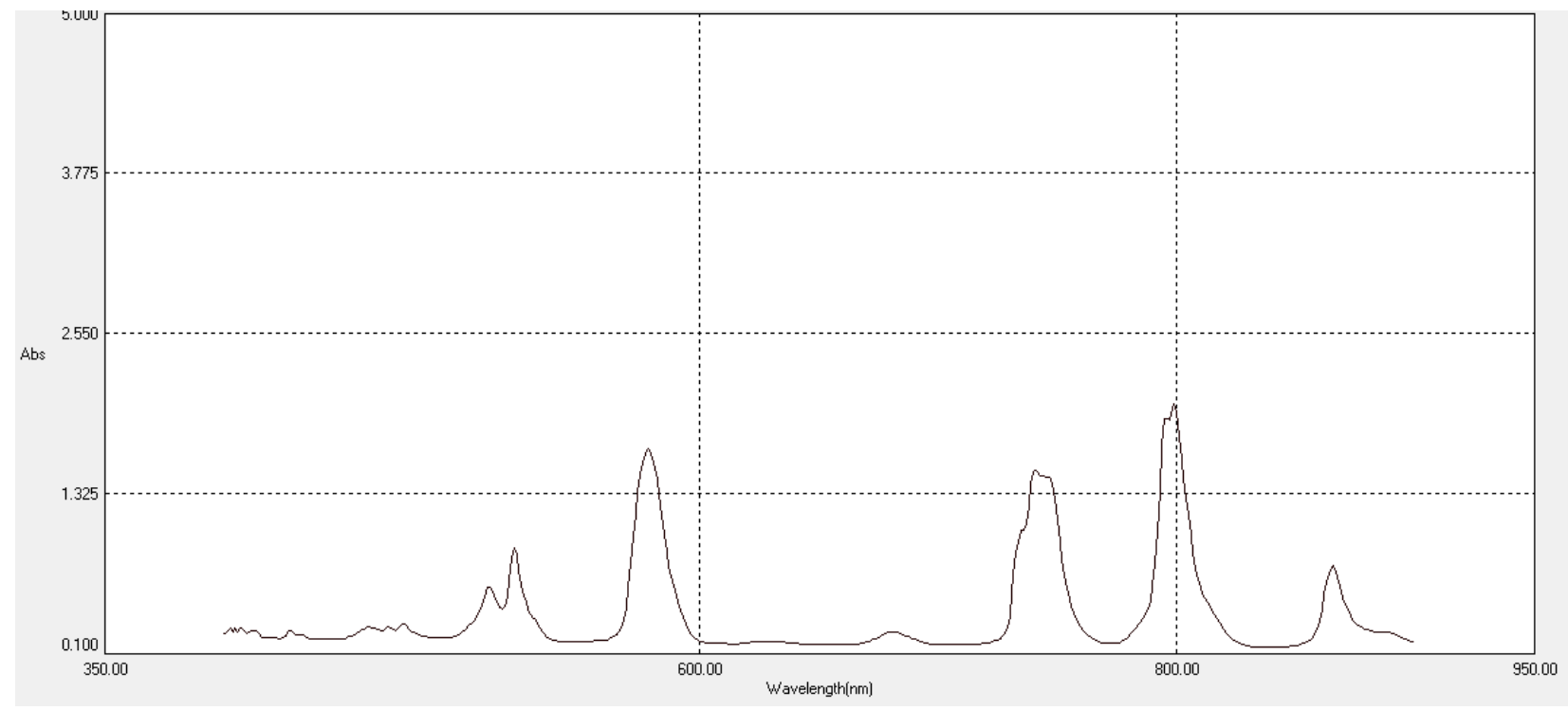

Graph 3: Absorption Spectra of Neodymium: Leucine: Urea in the Molar Ratio 1:1:2

\section{RESULTS AND DISCUSSION}

Bands of Neodymiumare intense as compared to their neighbouring metals. As the atomic number of Neodymium is even, therefore, according to Oddo-Harkins rule its bands are more intense as compared to odd atomic number nuclei. Forbidden Transitions are responsible for the Visible and near Infra-red spectra of Neodymium ternary complexes. There are some bands which are more intensified by magnitude as compared to others known as hypersensitive bands. In case of Neodymium, ground state of $\mathrm{Nd}^{+3}$ is $4 \mathrm{I}_{9 / 2}$. Ten bands of Neodymium are designated as $4 \mathrm{~F}_{3 / 2}, 4 \mathrm{~F}_{5 / 2}, 4 \mathrm{~F}_{7 / 2}, 4 \mathrm{~F}_{9 / 2}$, $4 \mathrm{G}_{5 / 2}, 4 \mathrm{G}_{7 / 2}, 4 \mathrm{G}_{9 / 2}, 2 \mathrm{G}_{9 / 2}, 4 \mathrm{G}_{11 / 2}$ and $2 \mathrm{P}_{1 / 2}$.In Nd ${ }^{+3}$, transition $4 \mathrm{I}_{9 / 2}$ to $4 \mathrm{G}_{5 / 2}$ is hypersensitive transition. Even minor co-ordination changes around the $\mathrm{Nd}(\mathrm{III})$, change the intensity too much. Some other bands also show large change in intensity but they do not obey normal selection rules. These transitions are known as Pseudo-hypersensitive transitions.

For all Neodymium complexes E1/ $\mathrm{E}_{3}$ lies between 10.10 and 10.14 and $\mathrm{E}_{2} / \mathrm{E}_{3}$ lies between 0.0500 and 0.0513 . If we change the ligand then there is small change in this ratio. In all the ternary complexes $F_{2}>F_{4}>F_{6}$, value of $F_{2}$ is largest. On complexation values of Slater-Condon parameters, Lande's parameter and Racah parameters decrease. This indicates expansion of the central metal ion orbital which further decreases the inter-atomic repulsion and spin orbit interaction. Hence R.M.S. deviation is small, which confirms that the formation of complex is perfect. On complexation, the value of Slater- Condon parameters decreases. The ratio of $\mathrm{F}_{4} / \mathrm{F}_{2}$ for Neodymium complexes lie in the range of 0.142 to 0.145 . The ratio of $F_{6} / F_{2}$ for Neodymium complexes is in the range of 0.01562 to 0.01578 . The ratio of $F 4 / F_{2}$ is nearly ten times as compared to $\mathrm{F}_{6} / \mathrm{F}_{2}$.

On complexation there is expansion of $4 \mathrm{f}$ orbital's which can be expressed in terms of Nephelauxetic Ratio ( $\beta$ ).Because of electron-electron repulsion and spin orbit interaction, Nephelauxetic ratio decreases and covalency increases. Due to shortening of metal-Ligand distance, Nephelauxetic ratio increases and hence Nephelauxetic ratio effects coordination number and overall geometry of complex. By knowing the value of Nephelauxetic ratio, bonding parameter is calculated. It has been observed that if ligands are the same and the solvent is the same then as atomic number increases, covalency increases. For Neodymium, it is ionic in nature. Covalency also increases with increase in concentration of amino-acid for same rare-earth. $\quad$ For Neodymium - Nephelauxetic Ratio, $\beta>1$. Therefore, $b^{1 / 2}$ is not real and $\delta=(1-\beta) / \beta$ 
is negative in this present work. Hence all ternary complexes of Neodymium make Ionic bonding with different amino-acids and with urea

\section{REFERENCES}

1. Carnall, W.T., Beitz, J.V., Crosswhite, H., Rajank, K.,\& Mann, J.B., (1983). Spectroscopic properties of the f-elements in compounds and solutions. D. Reidal Publishing Company, 389-450.

2. Gupta, A. K., \& Ujjwal, S. K., (2013). Optical study of Rare-Earth Lasing Material in Visible and Ir Region. Research Journal of Physical Sciences, 1(2), 9-14.

3. Adams, J. W., (1965). The Visible Region Absorption Spectra of Rare Earth Minerals. The American Mineralogist, 50, $356-$ 366.

4. Alaa, F.H., Muneer, A.A., \& Raghad, S.H., (2012). Study of liquid-liquid Extraction of Neodymium(III) by derivative of amino acid N-Acetylcysteine (NAC). Journal of Kerbala University, 10(10), 12-16.

5. Erskine, J.L., Blake, G.A., \& Flaten, C.J.,(1974).Optical Properties of Gd, Dy and Tb. Journal of The Optical Society of America, 64(10), 1332-1335.

6. Deepmala and Gokhroo, A.(2018).A comparative study of Absorption spectra of Neodymium in Glycine and Leucine as primary ligand and Urea as secondary ligand, International Journal of Research and Analytical Reviews, 5(3),596-600.

7. Ferraro, J.R., \& Becker, M., (1969). I.R Investigation of Several Rare-Earth Acetates \& Formates. Journal of Inorganic Chemistry, 32, 1495-1500.

8. Freeman, A.J., Dimmock, J.O., \& Watson, R.E., (1966). Fermi surface, Magnetic ordering and Electrical Properties of Rare Earth Metals. Physical Review Letters, 16 (3), 94-97.

9. Jain, S., Jain, Sushma., \& Dedar, R.,(2012). An absorption electronic spectral study for the interaction of Er(III) ion systems involving some biologically important ligands in DMF stereo medium, Journal of Applicable chemistry, 1(3), 376-380.

10. Judd, B.R., (1957). An Analysis of the absorption spectrum of praseodymium chloride. Royal Society, 241, 414-422.

11. Rodríguez, P., López, C. J., \& Hurtado, M. M. F. UV absorption processes in the debye sphere, by interaction plasma-metal on the surface of plant tissue Eugenia uniflora.

12. Judd, B.R., (1961). Optical Absorption Intensities of Rare-Earth Ions. Physical Review, 127, 750-760.

13. Kumar, P.A., \& Rao, T.R., (1997). Studies on some Rare-Earth metal complexes: Ten and six-coordinated complexes of an unsymmetrical Aminoacid derivative. Indian Acad. Science, 109, 89-97.

14. Narasimam, K.V., \& Girja, M.,(1969). Visible Absorption Spectrum of Neodymium in Calcium Fluoride \& Calcium Tungstate Lattices. Indian Journal of Pure and Applied Physics, 7, 427-430.

15. Deepmala, Gokhroo, A.(2018).A study of covalency of Erbium and Neodymium ternary complexes in Alanine and Urea, IOSR Journal of Applied Physics, 10(6),12-16.

16. Park, Chan-il., \& Cha, Ki-won.,(1999). Spectrometric Determination of Scandium (lll) in Monazite after Separation using Amberlite IRC718 Chelating Resin. Bulletin Korean Chemical Society, 20(12), 1409-1412.

17. Schuler, C. Chr., (1964). Recent Studies on the Optical Properties of Rare-Earth Metals, Phys. Letters, 12, 221-236.

18. Sengupta, D., \& Artman, J.O., (1970). Energy-Level Scheme for Nd3+ in LiYF4. Journal of Chemical Physics, 2, 1-9.

19. Gupta, A. K., \& Singhal, P. K. (2013). Microstrip antenna parameters improvement using EBG structutre. International 
journal of research in engineering and technology, 1(3), 35-40.

20. Surana, S.S.L., Mathur, R.C., Mehta, P.C., \& Tandon, S.P.,(1975). A Comparative Study of Strengths and Judd-Ofelt Intensity parameters of Various Nd3+ Complexes. Pure \& Applied Physics, 6, 363-365.

21. Tandon, S.P., \& Gupta, J.P.,(1970). A Note on Kubelka-Munk Theory of Diffuse Reflectance. Indian Journal of pure \& Applied Physics, 8, 231-232.

22. Tandon, S.P., \& Govil, R.C.,(1971). Diffuse Reflectance Spectra of some Nd3+ Complexes: Bonding. Spectroscopy Letters, $4(3 \& 4), 73-74$.

23. Evans, C.H., (1990). Biochemistry of Lanthanides, Chapter 4,Plenum Press, New York, USA.

24. Dimitrov, V., \& Komatsu, T.,(2005). Classification of Oxide Glasses-A Polarizabilty Approach. Journal of Solid State Chemistry, 178, 831-846.

25. Upase, A.B., \& Kalbende, P.P.,(2011). Spectrophotometric micro determination of Thorium(IV) and Uranium(VI) Chrome Azurol-S in the Presence of Cationic surfactant. E-Journal of chemistry, 8(3), 1132- 1141.

26. Guskos, N., Majszczyk, J., Typek, J., Zolnierkiewicz, G., Tomaszewicz E., \& Aidinis, K., (2010). Relative Intensities of f-f Transitions of Erbium(III) ion studied by photoacoustic spectroscopy. Rev.Adv.Mater.Science, 23, 97-101.

27. Othman, A. A., Osman, M. A., Wahdan, M. H., \& ABED-ELRAHIM, A. G. (2014). Thermal annealing and UV induced effects on the structural and optical properties of capping free ZnS nanoparticles synthesized by Co-precipitation method. International Journal of General Engineering and Technology, 3, 9-16.

28. Laegsgaard, J., (2002). Dissolution of rare-earth Clusters in siO2 by Aluminium Doping, A microscopic model. Physical Review B,65,174114

29. Ofelt, G.S., (1962). “Intensities of crystal spectra of rare-earth ions”. J. Chem. Phys. 37, 511-520.

30. Deepmala and Gokhroo,A.,(2018). Visible Absorption Spectra of Erbium and Neodymium in Arginine as a primary Ligand and Urea as a Secondary Ligands, Journal of Emerging Technologies and Innovative Research,5(10),399-406. 
